\title{
Ethnic, Race and Socioeconomic Disparities in Medulloblastoma: A Propensity Score-matched Analysis and Population-based Study
}

\section{Sihan Zhu}

Department of Neurosurgery and Neuro-Oncology, State Key Laboratory of Oncology in South China, Collaborative Innovation Center for Cancer Medicine, Sun Yat-sen University Cancer Center, Guangzhou, China

\section{Zhuqing Cheng}

Department of Neurosurgery and Neuro-Oncology, State Key Laboratory of Oncology in South China, Collaborative Innovation Center for Cancer Medicine, Sun Yat-sen University Cancer Center, Guangzhou, China

\section{Yanjiao Yu}

Department of Neurosurgery and Neuro-Oncology, State Key Laboratory of Oncology in South China, Collaborative Innovation Center for Cancer Medicine, Sun Yat-sen University Cancer Center, Guangzhou, China

\section{Ji Zhang}

Department of Neurosurgery and Neuro-Oncology, State Key Laboratory of Oncology in South China, Collaborative Innovation Center for Cancer Medicine, Sun Yat-sen University Cancer Center, Guangzhou, China

\section{Zhenghe Chen}

Department of Neurosurgery and Neuro-Oncology, State Key Laboratory of Oncology in South China, Collaborative Innovation Center for Cancer Medicine, Sun Yat-sen University Cancer Center, Guangzhou, China

\section{Chao Ke}

Department of Neurosurgery and Neuro-Oncology, State Key Laboratory of Oncology in South China, Collaborative Innovation Center for Cancer Medicine, Sun Yat-sen University Cancer Center, Guangzhou, China

\section{Qunying Yang}

Department of Neurosurgery and Neuro-Oncology, State Key Laboratory of Oncology in South China, Collaborative Innovation Center for Cancer Medicine, Sun Yat-sen University Cancer Center, Guangzhou, China

\section{Fuhua Lin}

Department of Neurosurgery and Neuro-Oncology, State Key Laboratory of Oncology in South China, Collaborative Innovation Center for Cancer Medicine, Sun Yat-sen University Cancer Center, Guangzhou, China

\section{Yinsheng Chen}

Department of Neurosurgery and Neuro-Oncology, State Key Laboratory of Oncology in South China, Collaborative Innovation Center for Cancer Medicine, Sun Yat-sen University Cancer Center, Guangzhou, China

\section{Jian Wang ( $\nabla$ wangjian2@sysucc.org.cn )}

Department of Neurosurgery and Neuro-Oncology, State Key Laboratory of Oncology in South China, Collaborative Innovation Center for Cancer Medicine, Sun Yat-sen University Cancer Center, Guangzhou, China https://orcid.org/00000002-9868-0491

\section{Research}

Keywords: Medulloblastoma. SEER, nomogram, Propensity score match, socioeconomic

Posted Date: January 28th, 2021

DOI: https://doi.org/10.21203/rs.3.rs-154336/v1 
License: (9) This work is licensed under a Creative Commons Attribution 4.0 International License. Read Full License 


\section{Abstract}

Background: Medulloblastoma (MB) is the most common malignant brain tumor of childhood. Studies have shown that the link between socio-economic status, morbidity and mortality from major cancers has changed significantly over the past 50 years, and therefore the socio-economic variation in cancer incidence is dynamic. Meanwhile, Socio-economic status (SES) characteristics of patients with medulloblastoma and the influence of Socio-economic status on their prognosis have been rarely discussed. Methods: Purpose of our study was aiming to understand ethnic, gender and socioeconomic disparities in medulloblastoma by using the SEER database. Propensity score matching of cases with controls by gender was conducted at the ratio of 1:1. Multivariate cox proportional hazards model was used to assess SES impact and clinically relevant variables of medulloblastoma specific death and overall survival. Independent prognostic factors determined by multivariate analysis were used to construct nomograms. Results: 2660 patients were enrolled after the matching. Study showed unemployed (MBSD, high level vs. low level, $H R=1.334)(O S$, high level vs. low level, $H R=1.311)$ and marital status $(O S$, married vs unmarried/unknow., $\mathrm{HR}=0.706)$ were important factors affecting the prognosis of medulloblastoma in male. Meanwhile, median household income (MBSD, Quartile 1 vs. Quartile 3, HR = 1.029) (OS, Quartile 1 vs. Quartile 2, HR $=1.809)(O S$, Quartile 1 vs. Quartile 3, HR = 1.592), residence (MBSD, urban vs. rural, $H R=0.414$ ) and insurance status (MBSD, insured vs. uninsured/unknow, $H R=0.566)(O S$, insured vs. uninsured/unknow, $H R=0.573)$ were significant factors affecting the prognosis of medulloblastoma in female. Through the calibration plot and C-index test, our nomogram was also of predictive significance. Conclusions: The unique features of medulloblastoma have provided a scenario for the analysis of the impact of racial, ethnic, gender and socioeconomic factors. The current findings have important public health implications for achieving the goal of a healthy population. Given the known morbidity rates and the long-term psychological, financial and medical burdens that these children and their families must bear, it is critical to identify and address these gaps.

\section{Introduction}

Medulloblastoma (MB) is the most common malignant brain tumor of childhood. Since medulloblastoma occurs in the posterior fossa, its symptoms are often vague and diagnosis may be delayed ${ }^{[1]}$. The differential diagnosis of medulloblastoma must be considered when parents detect a child with persistent symptoms such as nausea, vomiting, headache or other neurological disorders. Surgical resection is always the first line of treatment for medulloblastoma ${ }^{[2]}$. Despite great clinical progress of the treatment of medulloblastoma, there is still no cure for recurrence and metastasis, which is the leading cause of death in children ${ }^{[2]}$. In addition, the high cost of surgery and chemoradiotherapy bring a great challenge to the families of children, which seriously affect the prognosis of patients.

Monitoring and reducing health disparities based on socioeconomic status and ethnicity has long been important health policy goals in the whole wide world. Studies have shown that the link between socio-economic status(SES), morbidity and mortality from major cancers has changed significantly over the past 50 years, and therefore the socio-economic variation in

cancer incidence is dynamic ${ }^{[3]}$. The demographic characteristics of medulloblastoma have been reported several times in the previous literature. Demographically, there is no racial difference of the incidence of medulloblastoma. Among children, medulloblastoma is slightly more common to males, with a male-to-female incidence ratio of $0.63^{[4]}$. However, socioeconomic status characteristics of patients with medulloblastoma and the influence of SES on their prognosis have been rarely discussed.

In view of the current research situation, we developed some economic indicators to define SES on county-level. We would analyze SES factors on county-level in conjunction with common prognostic factors of medulloblastoma. In addition, the survival model was put in place to provide a basis of policy making and clinical decision-making.

\section{Method}

\section{Selection of Study Population}


The Surveillance, Epidemiology, and End Results (SEER) program of the National Cancer Institute collects and publishes cancer incidence, treatment and survival data from 18 population-based cancer registries, which cover more than $28 \%$ of the US population. Research data use-agreement was obtained from the SEER program (https://seer.cancer.gov/data) and data

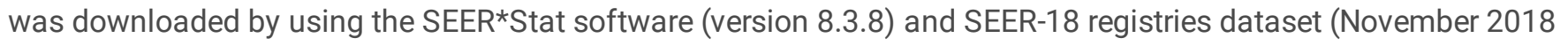
submission $)^{[5]}$. The exclusion criteria were as follows: (1) patients who were not histologically defined as medulloblastoma; (2) patients who had not complete follow-up records and survival status; (3) patients who had not complete SES index.

\section{Variables}

In our study, we included patients diagnosed with medulloblastoma patients between 1975 and 2016. Medulloblastoma patients were identified with the following selection algorithm: International Classification of Diseases for Oncology, Third Edition (ICD-0-3) histology codes of medulloblastoma. Data regarding patient demographics (race, age, gender, histology, tumor metastasis, surgery extent), state of residence, follow-up time, insurance, marital status, residence, SES factor, medulloblastoma specific death (MBSD) and overall survival (OS) were obtained. Estimates of SES were performed at the county-level and obtained from the US Census 2013-2017 American Community Survey 5-year data files ${ }^{[6]}$. For each variable, we use the median value to rank high and low levels to compare regional differences as described in Supplement Table 1. Marital status was classified as married and unmarried/unknow (including single, separated, divorced, widowed). Insurance status was characterized as insured (including medical care, military coverage, or private payers) and uninsured/unknow.

\section{Propensity score matching (PSM)}

Because medulloblastoma has obvious gender distribution, propensity score matching (PSM) was utilized to balance the clinical characteristics of male and female patients. Propensity scores were calculated using logistic regression and were based on age at diagnosis, race, histology, metastasis, surgery status, surgery extent, tumor size, education attainment, poverty, unemployed, crowding, language isolation, immigration, insurance status, marital status, residence and median household income. The nearest neighbor matching algorithm is adopted in order to achieve 1:1 matching, and the caliper is defined as $0.02^{[7]}$. Figure 1 showed the enrollment protocol.

\section{Statistical Analysis}

All statistical analyses were performed using R software, Version 3.6.2 (http://www.r-project.org) and SPSS software, Version 22.0 (SPSS Inc). Descriptive statistics were used to estimate baseline characteristics. Multivariate cox proportional hazards model was used to assess the SES impact and clinically relevant variables of MBSD and OS. Variables with P $<0.05$ were input into the multivariate cox proportional hazards model. Independent prognostic factors determined by multivariate analysis were used to construct nomograms for MBSD and $O S^{[8]}$. Calibration curves with 1000 resample bootstraps that compared nomogram-predicted versus actual observed survival probability. The predictions which fall on a $45^{\circ}$ diagonal line are expected to be a well-calibrated model. Consistency index (C-index) is used to evaluate the discriminatory effect of the index graph.

The $\mathrm{C}$-index ranges from 0.5 to 1.0, with a higher $\mathrm{C}$ index indicating a greater ability to differentiate between patients with different survival outcomes ${ }^{[9]}$.

\section{Result}

\section{Patient Demographics}

There were 3498 patients from the SEER database histologically classified as MB enrolled in this study. One patient did not have complete SES factors, and 39 patients did not have complete follow-up information and were excluded from the study. PSM analysis for medulloblastoma survival 
PSM was implemented to minimize selection bias between male and female. Before matching, significant differences were noted in race, unemployed and marital status between male and female MB patients. After PSM, there was no significant difference between male and female MB patients. Finally, 1330 paired MB cases were matched among male patients and female patients, respectively. The patient characteristics before and after matching were presented in Table 1. 
Table 1

Patient baseline characteristics before and after propensity score matching

\begin{tabular}{|c|c|c|c|c|c|c|c|c|}
\hline \multirow[b]{2}{*}{ Characteristics } & \multicolumn{4}{|c|}{$\begin{array}{l}\text { Before propensity score } \\
\text { matching }\end{array}$} & \multicolumn{4}{|c|}{$\begin{array}{l}\text { After propensity score } \\
\text { matching }\end{array}$} \\
\hline & Male & Female & Total & Pvalue & Male & Female & Total & Pvalue \\
\hline Total & $2128(61.5 \%)$ & 1330(39.5\%) & 3458 & & $1330(50.0 \%)$ & $1330(50.0 \%)$ & 2660 & \\
\hline Age at diagnosis & & & & 0.131 & & & & 0.487 \\
\hline Less than 4 & $520(60.8 \%)$ & $335(39.2 \%)$ & 855 & & $316(48.5 \%)$ & $335(51.5 \%)$ & 651 & \\
\hline 4 to 9 & $564(63.7 \%)$ & $322(36.3 \%)$ & 886 & & $339(51.3 \%)$ & $322(48.7 \%)$ & 661 & \\
\hline 10 to 19 & $432(63.9 \%)$ & $244(36.1 \%)$ & 676 & & $271(52.6 \%)$ & $244(47.4 \%)$ & 515 & \\
\hline 20 to 40 & $443(59.3 \%)$ & $304(40.7 \%)$ & 747 & & $293(49.1 \%)$ & $304(50.9 \%)$ & 597 & \\
\hline More than 40 & $169(57.5 \%)$ & $125(42.5 \%)$ & 294 & & $111(47.0 \%)$ & $125(53.0 \%)$ & 236 & \\
\hline Race & & & & 0.004 & & & & 0.183 \\
\hline White & $1794(62.3 \%)$ & 1086(37.7\%) & 2880 & & $1118(50.7 \%)$ & $1086(49.3 \%)$ & 2204 & \\
\hline Black & $146(51.6 \%)$ & 137 (48.4\%) & 283 & & 105 (43.4\%) & $134(56.6 \%)$ & 242 & \\
\hline API & 168 (64.4\%) & $93(35.6 \%)$ & 261 & & $91(49.5 \%)$ & 93 (50.5\%) & 184 & \\
\hline Al & $20(58.8 \%)$ & $14(41.2 \%)$ & 34 & & $16(53.3 \%)$ & $14(46.7 \%)$ & 30 & \\
\hline Histology & & & & 0.095 & & & & 0.529 \\
\hline Classic & $1861(62.2 \%)$ & $1129(37.8 \%)$ & 2990 & & $1148(50.4 \%)$ & $1129(49.6 \%)$ & 2277 & \\
\hline Desmoplastic & $204(56.7 \%)$ & 156 (43.3\%) & 360 & & 138 (46.9\%) & $156(53.1 \%)$ & 294 & \\
\hline Large cell & $63(58.3 \%)$ & $45(41.7 \%)$ & 108 & & $44(49.4 \%)$ & $45(50.6 \%)$ & 89 & \\
\hline Metastasis & & & & 0.692 & & & & 0.928 \\
\hline Yes & $1106(62.0 \%)$ & 677(38.0\%) & 1783 & & $672(49.8 \%)$ & $677(50.2 \%)$ & 1349 & \\
\hline No & $364(60.1 \%)$ & 242(39.9\%) & 606 & & $238(49.6 \%)$ & $242(50.4 \%)$ & 480 & \\
\hline Unknow & $658(61.6 \%)$ & $411(38.4 \%)$ & 1069 & & $420(50.5 \%)$ & $411(49.5 \%)$ & 831 & \\
\hline Surgery status & & & & 0.664 & & & & 0.806 \\
\hline Yes & $2006(61.6 \%)$ & $1249(38.4 \%)$ & 3255 & & $1252(50.1 \%)$ & $\begin{array}{l}1249 \\
(49.9 \%)\end{array}$ & 2501 & \\
\hline No & $122(60.1 \%)$ & $81(39.9 \%)$ & 203 & & $78(49.1 \%)$ & 81 (50.9\%) & 159 & \\
\hline Surgery extent & & & & 0.882 & & & & 0.783 \\
\hline $\begin{array}{l}\text { No } \\
\text { surgery/Unknow }\end{array}$ & 698(61.1\%) & $444(38.9 \%)$ & 1142 & & $450(50.3 \%)$ & $444(49.7 \%)$ & 894 & \\
\hline PTR & $356(60.5 \%)$ & $232(39.5 \%)$ & 588 & & $213(47.9 \%)$ & $232(52.1 \%)$ & 445 & \\
\hline STR & $347(61.7 \%)$ & $215(38.3 \%)$ & 562 & & $214(49.9 \%)$ & $215(50.1 \%)$ & 429 & \\
\hline GTR & $727(62.3 \%)$ & $439(37.7 \%)$ & 1166 & & $453(50.8 \%)$ & $439(49.2 \%)$ & 892 & \\
\hline Tumor size (cm) & & & & 0.120 & & & & 0.401 \\
\hline
\end{tabular}




\begin{tabular}{|c|c|c|c|c|c|c|c|c|}
\hline \multirow[b]{2}{*}{$\begin{array}{l}\text { Less than or equal } \\
4\end{array}$} & \multicolumn{4}{|c|}{$\begin{array}{l}\text { Before propensity score } \\
\text { matching }\end{array}$} & \multicolumn{4}{|c|}{$\begin{array}{l}\text { After propensity score } \\
\text { matching }\end{array}$} \\
\hline & $386(58.6 \%)$ & $273(41.4 \%)$ & 659 & & $274(47.5 \%)$ & $273(52.5 \%)$ & 520 & \\
\hline More than 4 & $522(63.8 \%)$ & $296(36.2 \%)$ & 818 & & $313(51.4 \%)$ & $296(48.6 \%)$ & 690 & \\
\hline Unknow & $1220(61.6 \%)$ & 761(38.4\%) & 1981 & & $770(50.3 \%)$ & 761(49.7\%) & 1531 & \\
\hline $\begin{array}{l}\text { Education } \\
\text { attainment }\end{array}$ & & & & 0.059 & & & & 0.351 \\
\hline High level & 1099(63.1\%) & $643(36.9 \%)$ & 1742 & & $619(49.0 \%)$ & $643(51.0 \%)$ & 1262 & \\
\hline Low level & $1029(60.0 \%)$ & $687(40.0 \%)$ & 1716 & & $711(50.9 \%)$ & $687(49.1 \%)$ & 1398 & \\
\hline Poverty & & & & 0.186 & & & & 0.816 \\
\hline Low level & $1086(62.6 \%)$ & $648(37.4 \%)$ & 1734 & & $642(49.8 \%)$ & $648(50.2 \%)$ & 1290 & \\
\hline High level & $1042(60.4 \%)$ & 682(39.6\%) & 1724 & & $688(50.2 \%)$ & $682(49.8 \%)$ & 1370 & \\
\hline Unemployed & & & & 0.005 & & & & 0.437 \\
\hline Low level & 1105(63.8\%) & $626(36.2 \%)$ & 1731 & & $606(49.2 \%)$ & $626(50.8 \%)$ & 1232 & \\
\hline High level & $1023(59.2 \%)$ & $704(40.8 \%)$ & 1727 & & $724(50.7 \%)$ & $704(49.3 \%)$ & 1428 & \\
\hline Crowding & & & & 0.923 & & & & 0.698 \\
\hline Low level & 1094(61.5\%) & $686(38.5 \%)$ & 1780 & & $676(49.6 \%)$ & $686(50.4 \%)$ & 1352 & \\
\hline High level & $1034(61.6 \%)$ & $655(38.4 \%)$ & 1678 & & $654(50.4 \%)$ & $655(49.6 \%)$ & 1298 & \\
\hline Language isolation & & & & 0.979 & & & & 0.587 \\
\hline Low level & $1129(61.6 \%)$ & $705(38.4 \%)$ & 1834 & & $691(49.5 \%)$ & $705(50.5 \%)$ & 1396 & \\
\hline High level & 999 (61.5\%) & $625(38.5 \%)$ & 1624 & & $639(50.6 \%)$ & $625(49.4 \%)$ & 1264 & \\
\hline Immigration & & & & 0.863 & & & & 0.786 \\
\hline Low level & $1096(61.7 \%)$ & 681(38.3\%) & 1777 & & $688(50.3 \%)$ & $681(49.7 \%)$ & 1369 & \\
\hline High level & $1032(61.4 \%)$ & 649(38.6\%) & 1681 & & 642 (49.7\%) & $649(50.3 \%)$ & 1291 & \\
\hline Insurance status & & & & 0.477 & & & & 0.423 \\
\hline Insured & $837(62.3 \%)$ & $507(37.7 \%)$ & 1344 & & $487(49.0 \%)$ & $507(51.0 \%)$ & 994 & \\
\hline Uninsured/Unknow & $1291(61.1 \%)$ & $823(38.9 \%)$ & 2114 & & $483(50.6 \%)$ & $823(49.4 \%)$ & 1666 & \\
\hline Marital status & & & & 0.019 & & & & 0.669 \\
\hline Married & $277(56.8 \%)$ & $211(43.2 \%)$ & 488 & & 203 (49.0\%) & $211(43.2 \%)$ & 414 & \\
\hline Unmarried/Unknow & $1851(62.3 \%)$ & $1119(37.7 \%)$ & 2970 & & $1127(50.2 \%)$ & $1119(37.7 \%)$ & 2246 & \\
\hline Residence & & & & 0.295 & & & & 0.722 \\
\hline Urban & 197 (62.9\%) & $116(37.1 \%)$ & 313 & & $115(49.8 \%)$ & $116(50.2 \%)$ & 231 & \\
\hline Rural & $1890(61.2 \%)$ & 1197(38.8\%) & 3087 & & $1193(49.9 \%)$ & $1197(50.1 \%)$ & 2390 & \\
\hline Unknow & $41(70.7 \%)$ & $17(29.3 \%)$ & 58 & & $22(56.4 \%)$ & $17(49.3 \%)$ & 39 & \\
\hline $\begin{array}{l}\text { Median household } \\
\text { income, US \$ }\end{array}$ & & & & 0.588 & & & & 0.996 \\
\hline
\end{tabular}




\begin{tabular}{|c|c|c|c|c|c|c|}
\hline \multirow[b]{2}{*}{$\begin{array}{l}\leq \text { Quartile } 1 \text { (US } \\
\$ 56310)\end{array}$} & \multicolumn{3}{|c|}{$\begin{array}{l}\text { Before propensity score } \\
\text { matching }\end{array}$} & \multicolumn{3}{|c|}{$\begin{array}{l}\text { After propensity score } \\
\text { matching }\end{array}$} \\
\hline & $528(61.0 \%)$ & $338(39.0 \%)$ & 866 & $338(50.0 \%)$ & $338(50.0 \%)$ & 676 \\
\hline $\begin{array}{l}\leq \text { Quartile } 2 \text { (US } \\
\$ 63250)\end{array}$ & $526(60.0 \%)$ & $351(40.0 \%)$ & 877 & $346(49.6 \%)$ & $351(50.4 \%)$ & 697 \\
\hline $\begin{array}{l}\text { SQuartile } 3 \text { (US } \\
\text { \$75730) }\end{array}$ & $530(62.4 \%)$ & $320(37.6 \%)$ & 850 & $321(50.1 \%)$ & $320(49.9 \%)$ & 641 \\
\hline $\begin{array}{l}\text { >Quartile } 4 \text { (US } \\
\$ 75740)\end{array}$ & $544(62.9 \%)$ & $321(37.1 \%)$ & 865 & $325(50.3 \%)$ & $321(49.7 \%)$ & 646 \\
\hline
\end{tabular}

\section{Impacts of SES factors on survival in different gender groups}

After excluding 39 cases without survival data, one case without SES information and PSM, we finally identified 2,660 cases for following analysis. The median follow-up of MB patients was 101 months. Kaplan-Meier curves using log-rank test were used to evaluate the effect of SES index on survival in patients with different genders. As showed in Fig. 2, male MB patients had higher MB-specific risk of death $(P=0.042)$ and overall risk of death $(P=0.031)$ in an area of high percentage of unemployment. Meanwhile, male MB patients had higher OS in an area of low percentage of immigration $(P=0.024)$. In female MB patients, SES on county-level appeared to have no significant effect on prolonging patients' OS or decreasing the MB-specific risk of death (Fig. 3).

\section{Impact of SES factors on MB patient outcomes}

The results of multivariate cox regression models in male MB patients were shown in Table 2. As we expected, Patients aged 4 to 9 years (vs age less than 4 years; hazard ratio $(\mathrm{HR})=0.654,95 \% \mathrm{Cl}=0.448-0.955, \mathrm{P}<0.001$ ), aged 10 to 19 years (vs aged less than 4 years; $(\mathrm{HR})=0.431,95 \% \mathrm{Cl}=0.293-0.634, \mathrm{P}<0.001)$, aged 20 to 40 years (vs aged less than 4 years; $(\mathrm{HR})=$ $0.419,95 \% \mathrm{Cl}=0.283-0.621, \mathrm{P}<0.001)$ and aged more than 40 years (vs aged less than 4 years; $(\mathrm{HR})=0.431,95 \% \mathrm{Cl}=$ $0.293-0.634, P=0.001)$ were significantly prolong male MB patients' OS. Meanwhile like reported before, desmoplastic medulloblastoma (MBSD: hazard ratio $(\mathrm{HR})=0.384,95 \% \mathrm{Cl}=0.242-0.611, \mathrm{P}<0.001)(\mathrm{OS}$ : hazard ratio $(\mathrm{HR})=0.383,95 \% \mathrm{Cl}$ $=0.246-0.595, \mathrm{P}<0.001)$ and large cell medulloblastoma (MBSD: hazard ratio $(\mathrm{HR})=0.243,95 \% \mathrm{Cl}=0.135-0.440, \mathrm{P}<0.001)$ (OS: hazard ratio $(\mathrm{HR})=0.298,95 \% \mathrm{Cl}=0.174-0.512, \mathrm{P}<0.001$ ) was associated with MBSD and OS. In terms of surgery, surgical excision can greatly improve MBSD (hazard ratio $(H R)=0.529,95 \% \mathrm{Cl}=0.350-0.798, \mathrm{P}=0.002$ ) and OS (hazard ratio $(\mathrm{HR})=0.537,95 \% \mathrm{Cl}=0.365-0.791, \mathrm{P}=0.002)$ in male patients with medulloblastoma. For every SES factor under study, we found that the more disadvantaged counties had lower relative OS and MBSD across all the SES factors studied, but the difference was only statistically significant in terms of unemployment (MBSD: hazard ratio $(\mathrm{HR})=1.334,95 \% \mathrm{Cl}=1.047-$ $1.701, \mathrm{P}=0.020)(\mathrm{OS}$ : hazard ratio $(\mathrm{HR})=1.311,95 \% \mathrm{Cl}=1.050-1.637, \mathrm{P}=0.017)$. It was interesting to note that we found unmarried was associated with better OS compared to married (hazard ratio $(\mathrm{HR})=0.706,95 \% \mathrm{Cl}=0.517-0.965, \mathrm{P}=0.029$ ).

We also had done multivariate cox regression models in female MB patients (Table 3). Like male MB patients, age at diagnosis, histology and surgical excision were favorable factor to MBSD and OS (Table 3). In addition to, insured status had significant influence on the female MB patients' MBSD (hazard ratio $(\mathrm{HR})=0.566,95 \% \mathrm{Cl}=0.397-0.807, \mathrm{P}=0.002$ ) and $0 \mathrm{~S}$ (hazard ratio $(\mathrm{HR})=0.573,95 \% \mathrm{Cl}=0.345-0.723, \mathrm{P}=0.002$ ). Interestingly, median household income was only an adverse factor for prolonging MBSD and OS in female MB patients. We found that women living in counties with high median household income had a worse prognosis (Table 3). 
Table 2

Multivariate cox proportional hazards regression models to predict MBSD and OS of medulloblastoma in male patients

\begin{tabular}{|c|c|c|c|c|}
\hline \multirow[t]{2}{*}{ Characteristics } & \multicolumn{2}{|l|}{ MBSD } & \multicolumn{2}{|l|}{ OS } \\
\hline & $\mathrm{HR}(95 \% \mathrm{Cl})$ & Pvalue & $\mathrm{HR}(95 \% \mathrm{Cl})$ & Pvalue \\
\hline \multicolumn{5}{|l|}{ Age at diagnosis } \\
\hline Less than 4 & Reference & - & Reference & - \\
\hline 4 to 9 & $0.950(0.607-1.486)$ & 0.823 & $0.654(0.448-0.955)$ & 0.028 \\
\hline 10 to 19 & $0.663(0.422-1.041)$ & 0.074 & $0.431(0.293-0.634)$ & $<.001$ \\
\hline 20 to 40 & $0.638(0.403-1.009)$ & 0.055 & $0.419(0.283-0.621)$ & $<.001$ \\
\hline More than 40 & $0.704(0.472-1.050)$ & 0.085 & $0.576(0.411-0.809)$ & 0.001 \\
\hline \multicolumn{5}{|l|}{ Race } \\
\hline White & Reference & - & Reference & - \\
\hline Black & $0.750(0.345-1.630)$ & 0.469 & $0.791(0.383-1.634)$ & 0.526 \\
\hline API & $0.971(0.421-2.241)$ & 0.945 & $1.008(0.461-2.203)$ & 0.985 \\
\hline Al & $0.616(0.258-1.470)$ & 0.275 & $0.641(0.285-1.442)$ & 0.232 \\
\hline \multicolumn{5}{|l|}{ Histology } \\
\hline Classic & Reference & - & Reference & - \\
\hline Desmoplastic & $0.384(0.242-0.611)$ & $<.001$ & $0.383(0.246-0.595)$ & $<.001$ \\
\hline Large cell & $0.243(0.135-0.440)$ & $<.001$ & $0.298(0.174-0.512)$ & $<.001$ \\
\hline \multicolumn{5}{|l|}{ Metastasis } \\
\hline Yes & Reference & - & Reference & - \\
\hline No & $0.888(0.523-1.508)$ & 0.661 & $1.081(0.660-1.769)$ & 0.758 \\
\hline Unknow & $1.504(0.876-2.582)$ & 0.139 & $1.755(1.060-2.907)$ & 0.029 \\
\hline \multicolumn{5}{|l|}{ Surgery status } \\
\hline Yes & Reference & - & Reference & - \\
\hline No & $0.529(0.350-0.798)$ & 0.002 & $0.537(0.365-0.791)$ & 0.002 \\
\hline \multicolumn{5}{|l|}{ Surgery extent } \\
\hline No surgery/Unknow & Reference & - & Reference & - \\
\hline PTR & $1.655(0.965-2.833)$ & 0.066 & $1.843(1.118-3.039)$ & 0.017 \\
\hline STR & $1.168(0.857-1.603)$ & 0.336 & $1.289(0.953-1.744)$ & 0.100 \\
\hline GTR & $1.349(0.921-1.976)$ & 0.135 & $1.479(1.044-2.178)$ & 0.470 \\
\hline \multicolumn{5}{|l|}{ Tumor size $(\mathrm{cm})$} \\
\hline Less than or equal 4 & Reference & - & Reference & - \\
\hline More than 4 & $1.029(0.743-1.424)$ & 0.856 & $1.172(0.845-1.627)$ & 0.341 \\
\hline
\end{tabular}




\begin{tabular}{|c|c|c|c|c|}
\hline \multirow[t]{2}{*}{ Characteristics } & \multicolumn{2}{|l|}{ MBSD } & \multicolumn{2}{|l|}{ OS } \\
\hline & $\mathrm{HR}(95 \% \mathrm{Cl})$ & Pvalue & $\mathrm{HR}(95 \% \mathrm{Cl})$ & Pvalue \\
\hline Unknow & $0.867(0.628-1.196)$ & 0.384 & $0.829(0.597-1.151)$ & 0.264 \\
\hline \multicolumn{5}{|l|}{ Education attainment } \\
\hline High level & Reference & - & Reference & - \\
\hline Low level & $0.968(0.821-1.499)$ & 0.767 & $1.097(0.831-1.447)$ & 0.513 \\
\hline \multicolumn{5}{|l|}{ Poverty } \\
\hline Low level & Reference & - & Reference & - \\
\hline High level & $1.138(0.776-1.670)$ & 0.507 & $1.009(0.708-1.438)$ & 0.961 \\
\hline \multicolumn{5}{|l|}{ Unemployed } \\
\hline Low level & Reference & - & Reference & - \\
\hline High level & $1.334(1.047-1.701)$ & 0.020 & $1.311(1.050-1.637)$ & 0.017 \\
\hline \multicolumn{5}{|l|}{ Crowding } \\
\hline Low level & Reference & - & Reference & - \\
\hline High level & $0.877(0.694-1.184)$ & 0.390 & $0.895(0.681-1.176)$ & 0.427 \\
\hline \multicolumn{5}{|l|}{ Language isolation } \\
\hline Low level & Reference & - & Reference & - \\
\hline High level & $0.806(0.571-1.134)$ & 0.220 & $0.840(0.613-1.151)$ & 0.278 \\
\hline \multicolumn{5}{|l|}{ Immigration } \\
\hline Low level & Reference & - & Reference & - \\
\hline High level & $0.819(0.646-1.037)$ & 0.098 & $0.819(0.659-1.018)$ & 0.072 \\
\hline \multicolumn{5}{|l|}{ Insurance status } \\
\hline Insured & Reference & - & Reference & - \\
\hline Uninsured/Unknow & $0.813(0.651-1.014)$ & 0.067 & $0.822(0.600-1.127)$ & 0.224 \\
\hline \multicolumn{5}{|l|}{ Marital status } \\
\hline Married & Reference & - & Reference & - \\
\hline Unmarried/Unknow & $0.794(0.552-1.140)$ & 0.211 & $0.706(0.517-0.965)$ & 0.029 \\
\hline \multicolumn{5}{|l|}{ Residence } \\
\hline Urban & Reference & - & Reference & - \\
\hline Rural & $1.286(0.578-2.286)$ & 0.538 & $1.069(0.516-2.213)$ & 0.859 \\
\hline Unknow & $1.068(0.529-2.227)$ & 0.824 & $0.939(0.492-1.791)$ & 0.849 \\
\hline \multicolumn{5}{|c|}{ Median household income, US \$ } \\
\hline$\leq$ Quartile 1 (US \$56310) & Reference & - & Reference & - \\
\hline 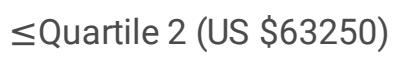 & $1.474(0.868-2.503)$ & 0.141 & $1.246(0.762-2.038)$ & 0.382 \\
\hline
\end{tabular}




\begin{tabular}{|lllll|}
\hline Characteristics & MBSD & \multicolumn{2}{l|}{ OS } & \\
\cline { 2 - 5 } & HR(95\%Cl) & Pvalue & HR(95\%Cl) & Pvalue \\
\hline SQuartile 3 (US \$75730) & $1.059(0.777-1.730)$ & 0.469 & $0.951(0.653-1.383)$ & 0.791 \\
\hline >Quartile 4 (US \$75740) & $1.067(0.776-1.476)$ & 0.689 & $1.050(0.786-1.402)$ & 0.742 \\
\hline
\end{tabular}


Table 3

Multivariate cox proportional hazards regression models to predict MBSD and OS of medulloblastoma in female patients

\begin{tabular}{|c|c|c|c|c|}
\hline \multirow[t]{2}{*}{ Characteristics } & \multicolumn{2}{|l|}{ MBSD } & \multicolumn{2}{|l|}{ OS } \\
\hline & $\mathrm{HR}(95 \% \mathrm{Cl})$ & Pvalue & $\mathrm{HR}(95 \% \mathrm{Cl})$ & Pvalue \\
\hline \multicolumn{5}{|l|}{ Age at diagnosis } \\
\hline Less than 4 & Reference & - & Reference & - \\
\hline 4 to 9 & $0.859(0.583-1.266)$ & 0.442 & $0.556(0.401-0.771)$ & $<.001$ \\
\hline 10 to 19 & $0.499(0.333-0.748)$ & 0.001 & $0.324(0.230-0.458)$ & $<.001$ \\
\hline 20 to 40 & $0.323(0.208-0.504)$ & $<.001$ & $0.219(0.155-0.320)$ & $<.001$ \\
\hline More than 40 & $0.517(0.361-0.739)$ & $<.001$ & $0.420(0.311-0.567)$ & $<.001$ \\
\hline \multicolumn{5}{|l|}{ Race } \\
\hline White & Reference & - & Reference & - \\
\hline Black & $0.750(0.303-1.856)$ & 0.534 & $0.678(0.313-1.467)$ & 0.324 \\
\hline API & $0.798(0.308-2.065)$ & 0.642 & $0.757(0.335-1.710)$ & 0.503 \\
\hline $\mathrm{Al}$ & $1.215(0.763-3.191)$ & 0.692 & $1.027(0.447-2.362)$ & 0.950 \\
\hline \multicolumn{5}{|l|}{ Histology } \\
\hline Classic & Reference & - & Reference & - \\
\hline Desmoplastic & $0.343(0.212-0.552)$ & $<.001$ & $0.304(0.198-0.467)$ & $<.001$ \\
\hline Large cell & $0.368(0.212-0.673)$ & $<.001$ & $0.302(0.183-0.468)$ & $<.001$ \\
\hline \multicolumn{5}{|l|}{ Metastasis } \\
\hline Yes & Reference & - & Reference & - \\
\hline No & $1.201(0.712-2.027)$ & 0.491 & $1.003(0.660-1.769)$ & 0.989 \\
\hline Unknow & $1.539(0.908-2.608)$ & 0.109 & $1.373(1.060-2.907)$ & 0.203 \\
\hline \multicolumn{5}{|l|}{ Surgery status } \\
\hline Yes & Reference & - & Reference & - \\
\hline No & $0.473(0.314-0.712)$ & $<.001$ & $0.499(0.345-0.723)$ & $<.001$ \\
\hline \multicolumn{5}{|l|}{ Surgery extent } \\
\hline No surgery/Unknow & Reference & - & Reference & - \\
\hline PTR & $1.596(0.938-2.715)$ & 0.085 & $1.490(0.915-2.426)$ & 0.109 \\
\hline STR & $1.089(0.775-1.528)$ & 0.624 & $1.084(0.493-1.482)$ & 0.612 \\
\hline GTR & $0.777(0.481-1.258)$ & 0.303 & $0.746(0.476-1.169)$ & 0.201 \\
\hline \multicolumn{5}{|l|}{ Tumor size $(\mathrm{cm})$} \\
\hline Less than or equal 4 & Reference & - & Reference & - \\
\hline More than 4 & $1.067(0.755-1.508)$ & 0.713 & $1.230(0.900-1.682)$ & 0.194 \\
\hline
\end{tabular}




\begin{tabular}{|c|c|c|c|c|}
\hline \multirow[t]{2}{*}{ Characteristics } & \multicolumn{2}{|l|}{ MBSD } & \multicolumn{2}{|l|}{ OS } \\
\hline & $\mathrm{HR}(95 \% \mathrm{Cl})$ & Pvalue & $\mathrm{HR}(95 \% \mathrm{Cl})$ & Pvalue \\
\hline Unknow & $1.161(0.811-1.663)$ & 0.414 & $1.313(0.941-1.830)$ & 0.109 \\
\hline \multicolumn{5}{|l|}{ Education attainment } \\
\hline High level & Reference & - & Reference & - \\
\hline Low level & $0.875(0.645-1.188)$ & 0.392 & $0.966(0.733-1.272)$ & 0.803 \\
\hline \multicolumn{5}{|l|}{ Poverty } \\
\hline Low level & Reference & - & Reference & - \\
\hline High level & $1.202(0.800-1.805)$ & 0.376 & $1.245(0.863-1.796)$ & 0.240 \\
\hline \multicolumn{5}{|l|}{ Unemployed } \\
\hline Low level & Reference & - & Reference & - \\
\hline High level & $1.125(0.875-1.447)$ & 1.125 & $1.155(0.920-1.451)$ & 0.214 \\
\hline \multicolumn{5}{|l|}{ Crowding } \\
\hline Low level & Reference & - & Reference & - \\
\hline High level & $1.029(0.762-1.390)$ & 0.850 & $0.972(0.744-1.269)$ & 0.833 \\
\hline \multicolumn{5}{|l|}{ Language isolation } \\
\hline Low level & Reference & - & Reference & - \\
\hline High level & $1.170(0.824-1.661)$ & 0.379 & $1.087(0.797-1.484)$ & 0.597 \\
\hline \multicolumn{5}{|l|}{ Immigration } \\
\hline Low level & Reference & - & Reference & - \\
\hline High level & $1.079(0.853-1.366)$ & 0.527 & $1.052(0.849-1.303)$ & 0.644 \\
\hline \multicolumn{5}{|l|}{ Insurance status } \\
\hline Insured & Reference & - & Reference & - \\
\hline Uninsured/Unknow & $0.566(0.397-0.807)$ & 0.002 & $0.573(0.345-0.723)$ & 0.001 \\
\hline \multicolumn{5}{|l|}{ Marital status } \\
\hline Married & Reference & - & Reference & - \\
\hline Unmarried/Unknow & $0.915(0.655-1.278)$ & 0.602 & $0.788(0.591-1.049)$ & 0.103 \\
\hline \multicolumn{5}{|l|}{ Residence } \\
\hline Urban & Reference & - & Reference & - \\
\hline Rural & $0.414(0.178-0.966)$ & 0.041 & $0.670(0.309-1.451)$ & 0.310 \\
\hline Unknow & $0.735(0.345-1.566)$ & 0.425 & $0.990(0.489-2.006)$ & 0.979 \\
\hline \multicolumn{5}{|c|}{ Median household income, US \$ } \\
\hline$\leq$ Quartile 1 (US \$56310) & Reference & - & Reference & - \\
\hline$\leq$ Quartile 2 (US \$63250) & $1.593(0.930-2.729)$ & 0.090 & $1.808(1.111-2.943)$ & 0.017 \\
\hline
\end{tabular}




\begin{tabular}{|lllll|}
\hline Characteristics & MBSD & \multicolumn{2}{l|}{ OS } \\
\cline { 2 - 5 } & HR(95\%Cl) & Pvalue & HR(95\%Cl) & Pvalue \\
\hline SQuartile 3 (US \$75730) & $1.029(0.005-2.281)$ & 0.047 & $1.592(1.098-2.308)$ & 0.014 \\
\hline >Quartile 4 (US \$75740) & $1.170(0.745-1.446)$ & 0.826 & $1.127(0.835-1.520)$ & 0.345 \\
\hline
\end{tabular}

\section{Construction and validation of nomograms for MBSD and OS}

Then, we utilized a nomogram to construct a visual survival model to predict the prognosis of MB patients. Figures 4 showed important prognostic factors for MBSD and OS at 3-, 5-, and 10- years of MB patients. MB patients in SEER dataset were randomly divided to a training cohort $(n=1862)$ for nomogram construction and a validation cohort $(n=798)$ for validation in a 7-to-3 ratio. The C-index for MBSD prediction in training cohort and validation cohort was 0.641 and 0.657 , respectively. Meanwhile, the C-index for OS prediction in training cohort and validation cohort was 0.650 and 0.657 , respectively. Calibration plots for the probability of MBSD and OS at 3-, 5- and 10-years demonstrated an agreement between the predictive nomogram and actual observation (Fig. 5) (Fig. 6).

\section{Discussion}

Medulloblastoma (MB) is the most common embryonic tumor of the central nervous system, especially among children. Largely giving the credit to technology improvements in diagnosis and risk stratification which now integrate histology classification and molecular classification, MB has become one of the most rapidly advancing tumors in diagnosis and risk stratification $^{[10]}$. Despite these advances, the prognosis of MB patients has not been proven to be related to gender, race, ethnicity and unfavorable social environment such as low household income, low educational attainment, crowding, language isolation and being part of a new immigrant community ${ }^{[11]}$. The initial aim of our study, therefore, was to understand these differences and address the issues that should be brought to the attention of health policy makers.

Brain tumors are a major disease that affects a large proportion of the population. At the same time, the brain tumor treatment environment and hospital facilities have put forward great demands, including high-resolution MRI, use of intraoperative monitoring and image guidance, and dedicated postoperative neurocritical ICU. This made the regions which were in poor SES and poor healthcare system could not offer an effective and standardized treatment for patients with brain tumors $^{[12]}$. In terms of brain tumors, many studies have shown that SES factors were associated with the prognosis of patients. Previous analysis has found a significant link between improved socioeconomic status and glioma, which could lead to different racial or ethnic differences in incidence ${ }^{[13]}$. QT Ostrom et al' study found that non-Hispanic whites who were diagnosed with glioblastoma had a poorer prognosis than the rest of the population ${ }^{[14]}$. This pattern was found in both the era analysis before and after the STUPP protocol in patients receiving post-excision treatment ${ }^{[14]}$. The potential impact of SES on cancer survival has been investigated for different tumor types in several countries, and community poverty levels have repeatedly been associated with cancer mortality ${ }^{[15]}$. Higher SES was associated with greater likelihood of surgical resection and improved survival in patients with ovarian cancer ${ }^{[16]}$. In Finland, the incidence of colon cancer in men with elementary education attainment has a steep increase ${ }^{[17]}$. In China, SES is an important indicator of thyroid cancer in Hangzhou, and there are spatial differences in its influence ${ }^{[18]}$.

In terms of gender, there had already reported that MB incidence was significantly higher in male than in female (about $60 \%$ male $)^{[19]}$. Meanwhile, there were also differences in gender ratios among different molecular subtypes. However, the underlying relation of gender on the occurrence and prognosis of medulloblastoma has not been clearly studied. Although population-based studies are few, there does not appear to be any substantial difference in MB incidence across ethnic or geographic areas ${ }^{[20]}$. Our cohort, obtained from the SEER database, had a larger sample size and was widely distributed, 
which reinforced the representation of individuals with medulloblastoma. Meanwhile, we used PSM to eliminate selection bias, leaving no difference in the baseline data we studied. Consistent with previous results, our multivariate analysis demonstrated that age was a significant factor affecting the prognosis of medulloblastoma in both male and female. For example, WNT-MB tumors rarely metastasized at the time of diagnosis, and patients younger than 16 years had a good prognosis, with $95 \%$ patients surviving for more than 5 years. In contrast, adults with WNT-MB may have poorer outcomes ${ }^{\text {[20] }}$ The main reason is that the presence or absence of different molecular, genetic and clinical characteristics is closely related to prognosis. However, infant, childhood and adult MBs represent clinically and biologically distinct groups. Our results also failed to demonstrate an association between the prognosis of medulloblastoma and race. To date, germline mutations in six genes have been associated with a significantly increased risk of medulloblastoma. Nevertheless, germline mutation is closely related to family heredity and population. When genomic research is conducted, it may be necessary to redefine the differences in outcome of ethnic traditions ${ }^{[21]}$. Yang et al ${ }^{[22]}$ observed that genomic variants co-isolated with native American ancestry were associated with a risk of recurrence of acute lymphoblastic leukemia, even after adjusting for known prognostic factors. Whether the same phenomenon applies to embryonic tumors, such as medulloblastoma, is unclear. Therefore, we could try to interrogate genome-wide germline single-nucleotide poly morphisms in a cohort of patients with MB to investigate the relationship between germline and race.

Survival inequality caused by socioeconomic factors, namely, educational attainment, poverty, unemployed, crowding, language isolation, immigration, insurance status, marital status, residence and median household income, has been well documented for many cancer types in literature ${ }^{[23]}$. Our multivariate analysis demonstrated unemployed and marital status were important factors affecting the prognosis of medulloblastoma in male. Meanwhile, median household income, residence and insurance status were significant factors affecting the prognosis of medulloblastoma in female. As our study reflected, the downstream mechanisms through which SES influences medulloblastoma are not fully understood. But there is no denying that a country's SES is closely related to the establishment of the medical social security system. In addition to, our results do reflect that insurance status is associated with MB prognosis, regardless of gender. Our findings highlighted the potential gap between recommendations and the reality of access to primary health care, which may be even greater for more

vulnerable populations ${ }^{[24]}$. People living in relatively backward social and economic conditions were more vulnerable to being affected by MB. Children with limited support who face language, insurance, and financial barriers, and as a high incidence of $\mathrm{MB}$, are at the greater risk for treatment failure. Last but not least, even if the child can be treated surgically, the following chemoradiotherapy also posed a great challenge to the economic conditions of the patients' families. Therefore, our study was not only focused on the impact of SES on MB. Moreover, we hoped to provide dedicated analyses and intervention advice to MB diagnosis and treatment through the establishment of a risk model.

\section{Strengths And Limitations}

The strengths of the current study included the nearly universal representation of medulloblastoma from the US population over multiple years. To the best of our knowledge, the current study was the first of its kind to examine MBSD and OS of medulloblastoma by SES, and one of the few to stratify by ethnicity, insurance status, marital status, residence and median household income. In addition, we utilized PSM to make all baseline data non-different, making the results more convincing. Finally, we set up a nomogram to visualize the prognosis of SES in patients with MB.

Meanwhile, there were several limitations to the current study. Our SES factors were reflected on county-level rather than on individual-level. Thus, we used classification method from the more influential literature to classify socioeconomic classifications ${ }^{[15]}$. Secondly, if used as a model to predict patient outcomes only, our prediction model is more focused on finding SES factors that affect the prognosis of MB. Therefore, there is a lack of information such as molecular subgroup, chemotherapy and radiotherapy, which were critical to the prognosis of MB patients. Besides, cancer registry data represent the most complete set of data describing cancer incidence and survival patterns, but this type of data has a few inherent limitations, including known problems with the integrity of treatment information ${ }^{[25]}$. In addition, our results needed to be interpreted in the context of the limitations inherent in using SEER registry and census data and extrapolating regionally

Page $15 / 22$ 
based indicators to investigate the social determinants of health of this particular population ${ }^{[15]}$. Therefore, more prospective studies were needed to evaluate the individual impact of socioeconomic indicators on MB patients.

\section{Conclusions}

The unique features of medulloblastoma have provided a scenario for the analysis of the impact of racial, ethnic, gender and socioeconomic factors. To our knowledge, these incidence estimates are based on population, including about $99.9 \%$ of the U.S. population, and SEER data cover about $28 \%$ of the population ${ }^{[26]}$. The current findings have important public health implications for achieving the goal of a healthy population because socio-economic and therapeutic factors can be changed through public health and clinical interventions. Given the known morbidity rates and the long-term psychological, financial and medical burdens that these children and their families must bear, it is critical to identify and address these gaps.

\section{Declarations}

\section{ETHICS APPROVAL AND CONSENT TO PARTICIPATE}

Not applicable.

\section{CONSENT FOR PUBLICATION}

Not applicable.

\section{FUNDING}

Not applicable.

\section{AUTHORS' CONTRIBUTIONS}

SZ, ZC and YY conceived, designed, analyzed the data, and wrote the manuscript. JW, YC, and FL conceptualized and developed an outline for the manuscript. ZC, JZ, CK and QY made a review of the manuscript. All authors contributed to the article and approved the submitted version.

\section{CONFLICT OF INTEREST}

There are no conflicts of interest to disclose.

\section{ACKNOWLEDGEMENTS}

Not applicable.

\section{DATA AVAILABILITY STATEMENT}

The datasets generated and analyzed during this study are available from Surveillance, Epidemiology, and End Results (SEER) Program (www.seer.cancer.gov) SEER ${ }^{\circ}$ Stat Database: Incidence-SEER 18 Regs Custom Data (with additional treatment fields), Nov 2018 Sub (1975-2016 varying), National Cancer Institute, DCCPS, Surveillance Research Program, released April 2019, based on the November 2018 submission.

\section{References}

1. Quinlan, A; Rizzolo, D, Understanding medulloblastoma. Journal of the American Academy of Physician Assistants: October 2017-Volume 30-Issue 10-p30-36. 
2. Miranda Kuzan-FC, Juraschka K, Taylor MD. Medulloblastoma in the Molecular Era. J Korean Neurosurg Soc. 2018;61(3):292-301.

3. Singh GK, Miller BA, Hankey BF, et al. Changing area socioeconomic pattern in U.S. cancer mortality, 1950-1998: Part Iall cancers among men. Journal of the National Cancer Institute. 2002;94(12):904-915.

4. Millard NE, De Braganca KC. Medulloblastoma. J Child Neurol. 2016;31(12):1341-1353.

5. National Cancer Institute. Surveillance, Epidemiology, and End Results (SEER) Program (www.seer.cancer.gov) SEER*Stat Database: Incidence-SEER 18 Regs Custom Data (with additional treatment fields), Nov 2018 Sub (1975-2016 varying), National Cancer Institute, DCCPS, Surveillance Research Program, released April 2019, based on the November 2018 submission.

6. Gray RJ. A class of k-sample tests for comparing the cumulative incidence of a competing risk. Ann Stat. 1988;16:11411154.

7. Austin PC. Balance diagnostics for comparing the distribution of baseline covariates between treatment groups in propensity-score matched samples. Stat Med. 2009;28(25):3083-3107.

8. Ji P, Gong Y, Jiang CC, et al. Association between socioeconomic factors at diagnosis and survival in breast cancer: A population-based study. Cancer Med. 2020;9(5):1922-1936.

9. Wolbers M, Koller MT, Witteman JC, et al. Prognostic models with competing risks: methods and application to coronary risk prediction. Epidemiology. 2009;20:555-561.

10. Orr BA. Pathology, diagnostics, and classification of medulloblastoma. Brain Pathol. 2020;30(3):664-678.

11. Truong B, Green AL, Friedrich P, et al. Ethnic, Racial, and Socioeconomic Disparities in Retinoblastoma. JAMA Pediatr.2015;169(12):1096-1104.

12. Helal AE, Abouzahra $H$, Fayed AA, et al. Socioeconomic restraints and brain tumor surgery in low-income countries. Neurosurg Focus. 2018 Oct;45(4):E11.

13. Porter AB, Lachance DH, Johnson DR. Socioeconomic status and glioblastoma risk: a population-based analysis. Cancer Causes Contro 2015;26(2):179-185.

14. Ostrom QT, Cote DJ, Ascha M, et al. Adult Glioma Incidence and Survival by Race or Ethnicity in the United States From 2000 to 2014. JAMA Oncol. 2018 Sep 1;4(9):1254-1262.

15. Fleisch Marcus A, Illescas AH, Hohl BC, et al. Relationships between social isolation, neighborhood poverty, and cancer mortality in a population-based study of US adults. PLoS One. 2017;12: e0173370.

16. Gardy J, Dejardin O, Thobie A, et al. Impact of socioeconomic status on survival in patients with ovarian cancer. Int J Gynecol Cancer. 2019 May;29(4):792-801.

17. Savijärvi S, Seppä K, Malila N, , et al. Trends of colorectal cancer incidence by education and socioeconomic status in Finland. Acta Oncol. 2019 Nov;58(11):1557-1563.

18. Fei X, Lou Z, Christakos G, , et al. Contribution of industrial density and socioeconomic status to the spatial distribution of thyroid cancer risk in Hangzhou, China. Sci Total Environ. 2018 Feb 1;613-614:679-686.

19. Peris-Bonet R, Martinez-Garcia C, Lacour B, et al. Childhood central nervous system tumours-incidence and survival in Europe (1978-1997): report from Automated Childhood Cancer Information System project. Eur J Cancer. 2006;42:2064-80.

20. Remke, M. et al. Adult medulloblastoma comprises three major molecular variants. J Clin Oncol. 29, 2717-2723 (2011).

21. Lim JY-S, Bhatia S, Robison LL, et al. Genomics of racial and ethnic disparities in childhood acute lymphoblastic leukemia. Cancer. 2014;120(7):955-962.

22. Yang JJ, Cheng $C$, Devidas $M$, et al. Ancestry and pharmacogenomics of relapse in acute lymphoblastic leukemia. Nat Genet. 2011;43(3):237-241.

23. Bray F, Ferlay J, Soerjomataram I, Siegel RL, et al. Global cancer statistics 2018: GLOBOCAN estimates of incidence and mortality worldwide for 36 cancers in 185 countries. CA Cancer J Clin. 2018;68(6):394-424. 
24. Dougherty D, Chen X, Gray DT, et al. Child and adolescent health care quality and disparities: are we making progress ? Acad Pediatr. 2014;14(2): 137-148.

25. Noone AM, Lund JL, Mariotto A, et al. Comparison of SEER treatment data with Medicare claims. Med Care. 2016;54(9):e55-e64.

26. Jemal A, Ward EM, Johnson CJ, et al. Annual report to the nation on the status of cancer, 1975-2014, featuring survival. $J$ Natl Cancer Inst. 2017;109(9).

\section{Figures}

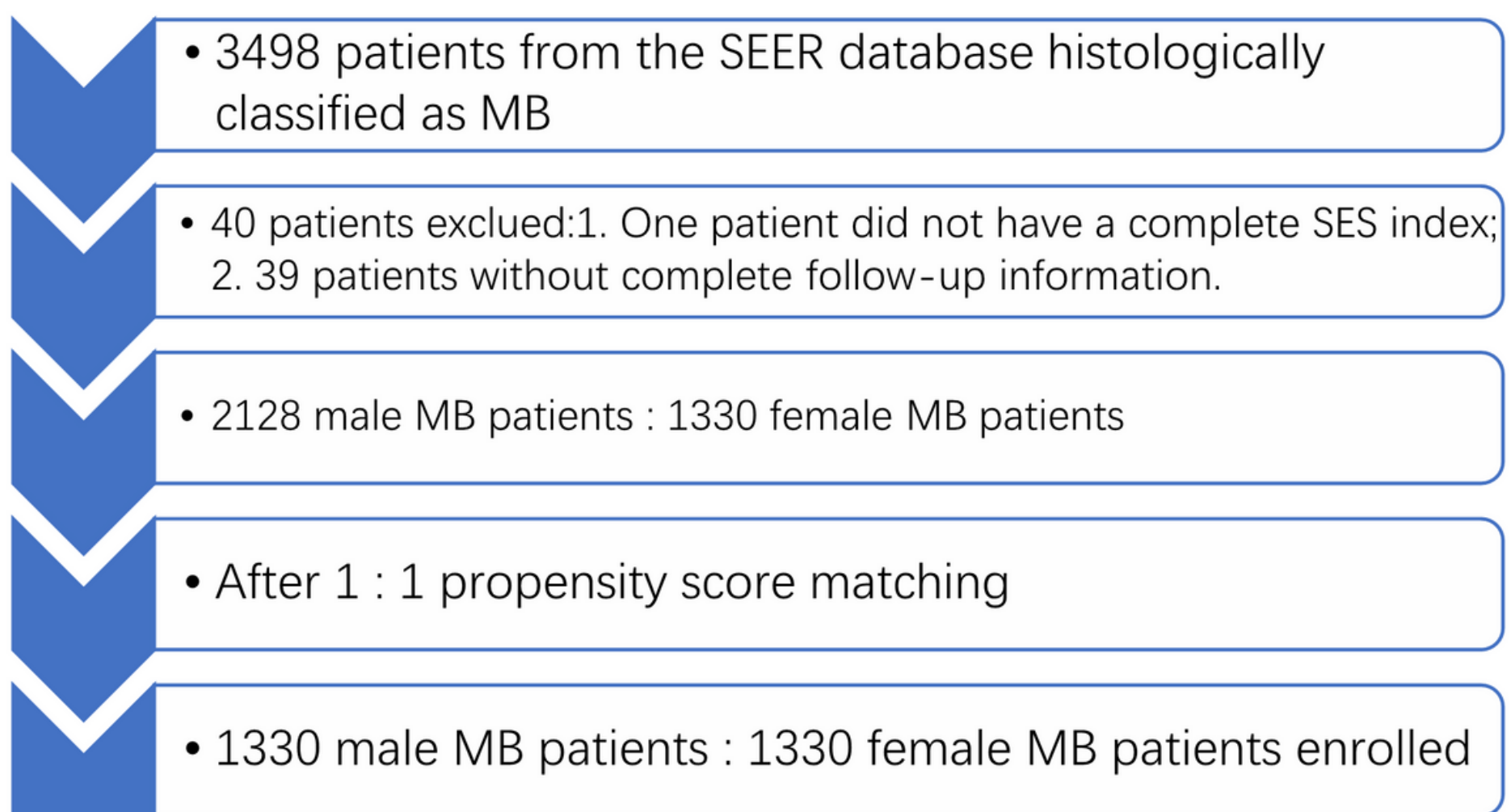

Figure 1

Flow diagram showing inclusion and exclusion criteria. 
Medulloblastoma specific death in male patients:

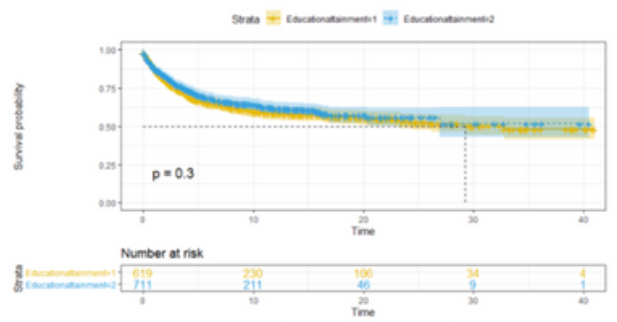

Education attainment
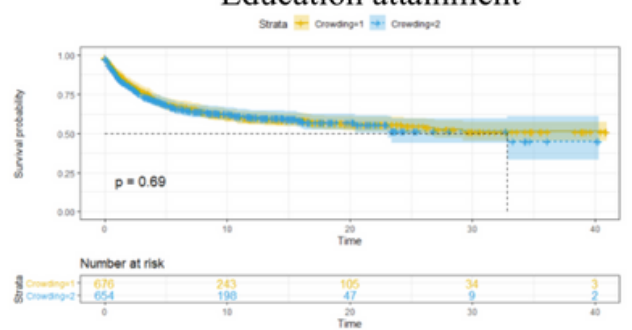

Crowding
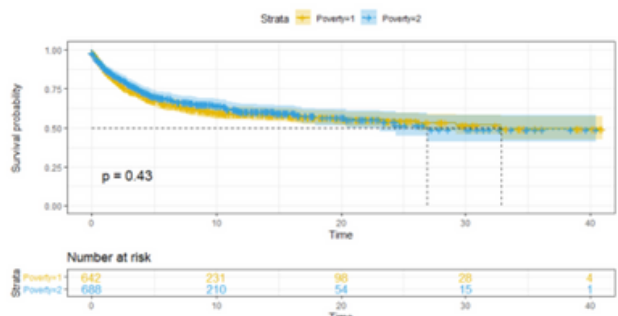

Poverty

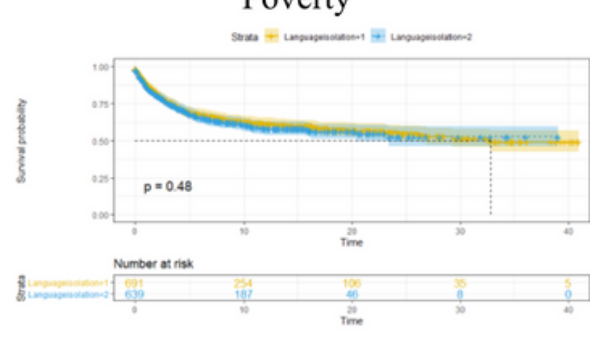

Language isolation
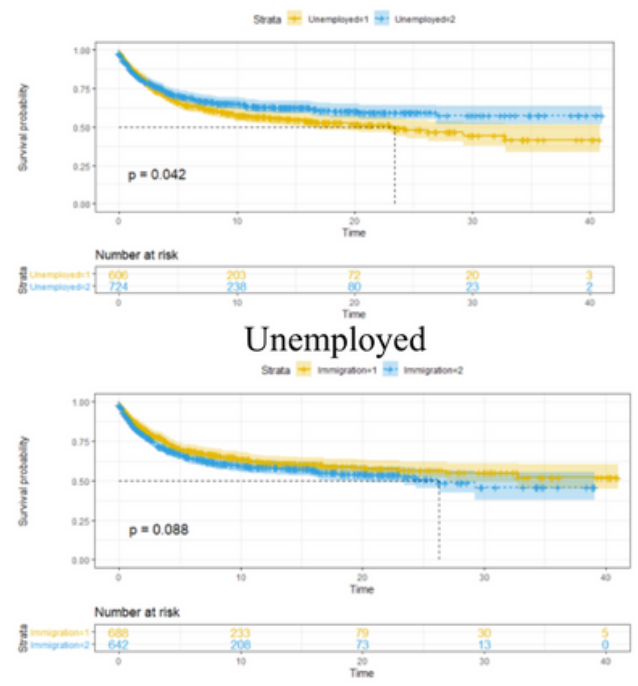

Immigration

Overall Survival in male patients:

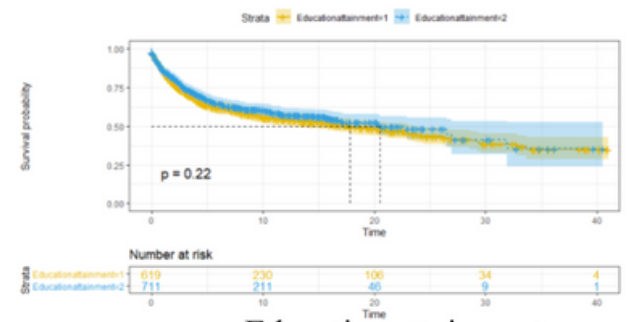

Education attainment
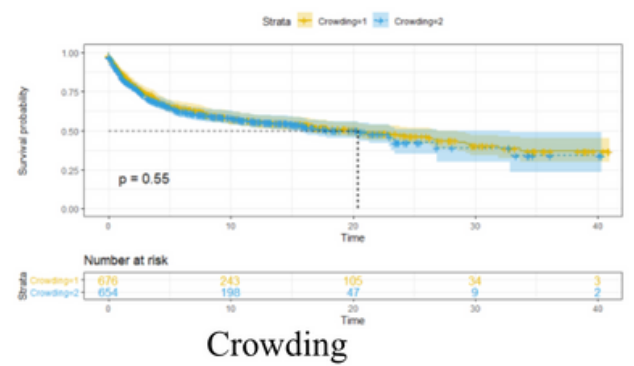

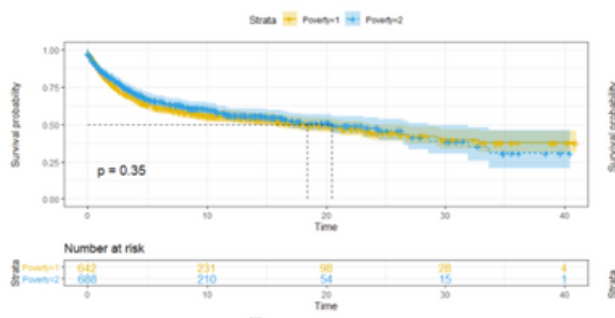

Poverty
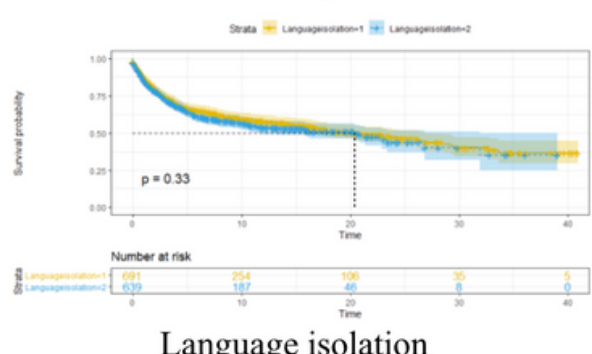

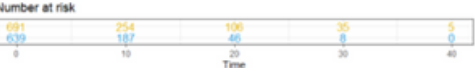

Language isolation

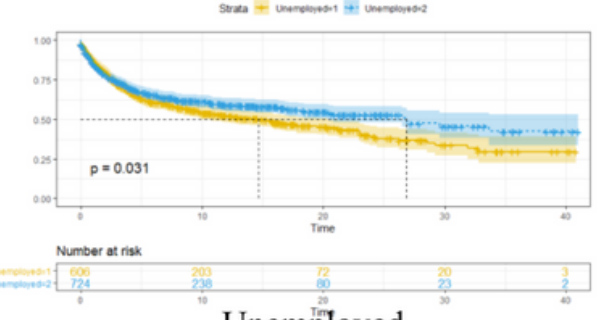

Unemployed
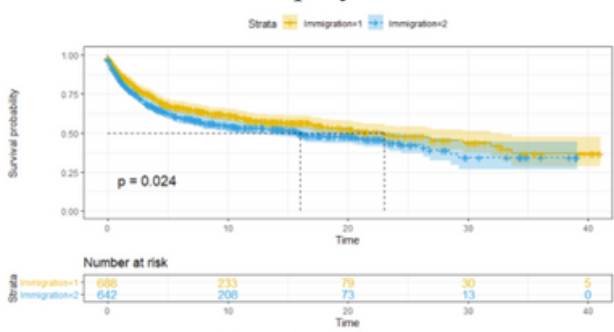

Immigration

\section{Figure 2}

Kaplan-Meier survival curves by socio-economic status on county-level in male patients. (1=high level; $2=$ low level) 
Medulloblastoma specific death in female patients

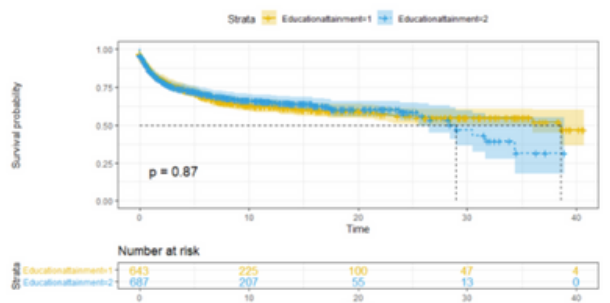

Education attainment



Overall Survival in female patients

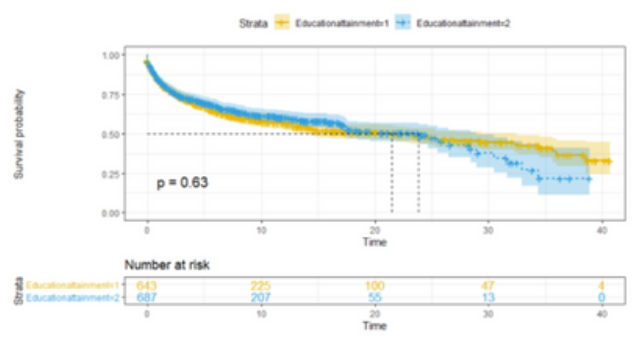

Education attainment
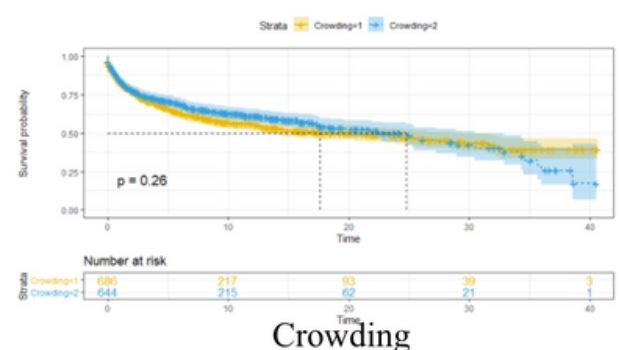

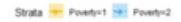


Language isolation
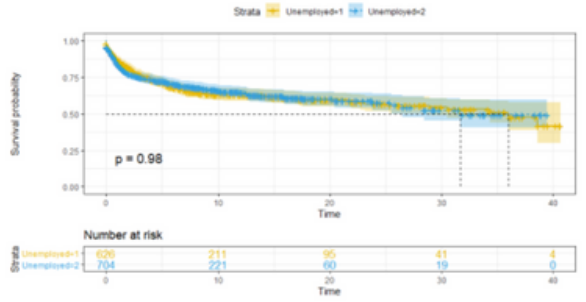

Unemployed
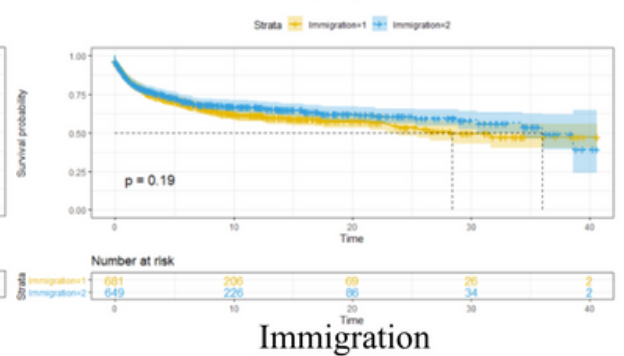

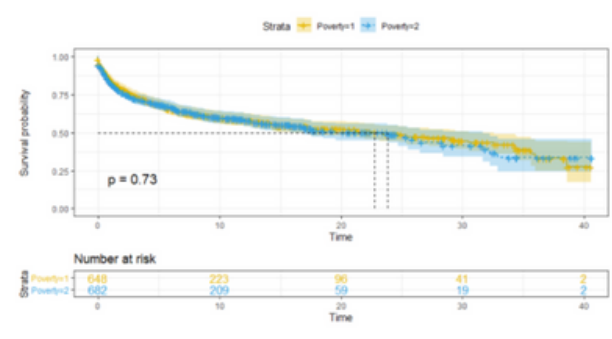

Poverty

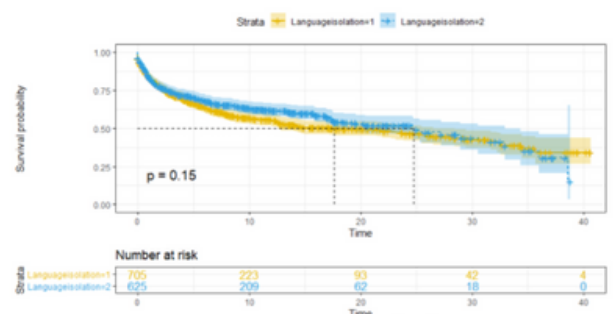

Language isolation

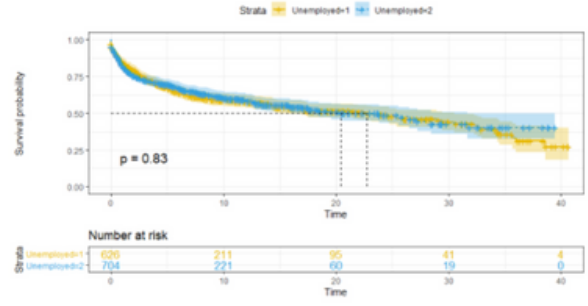

Unemployed

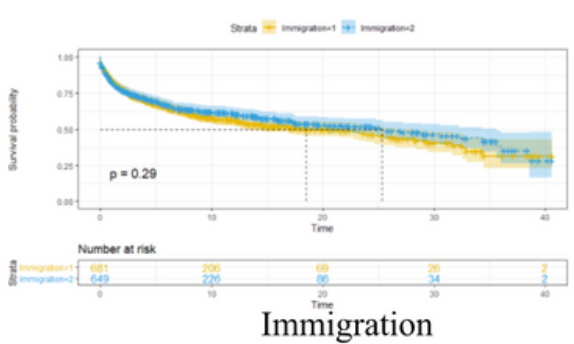

\section{Figure 3}

Kaplan-Meier survival curves by socio-economic status on county-level in female patients.(1=high level; $2=$ =low level) 
Educatonataimmert

Povery

Unemelobed

Medianhousenoldincome

cromdino

Langugaceisolaton

Immigration

insuancestatus

Martakstatus

Residence

Total Points

3year survival

5 year sumval

10-year sunvival

B
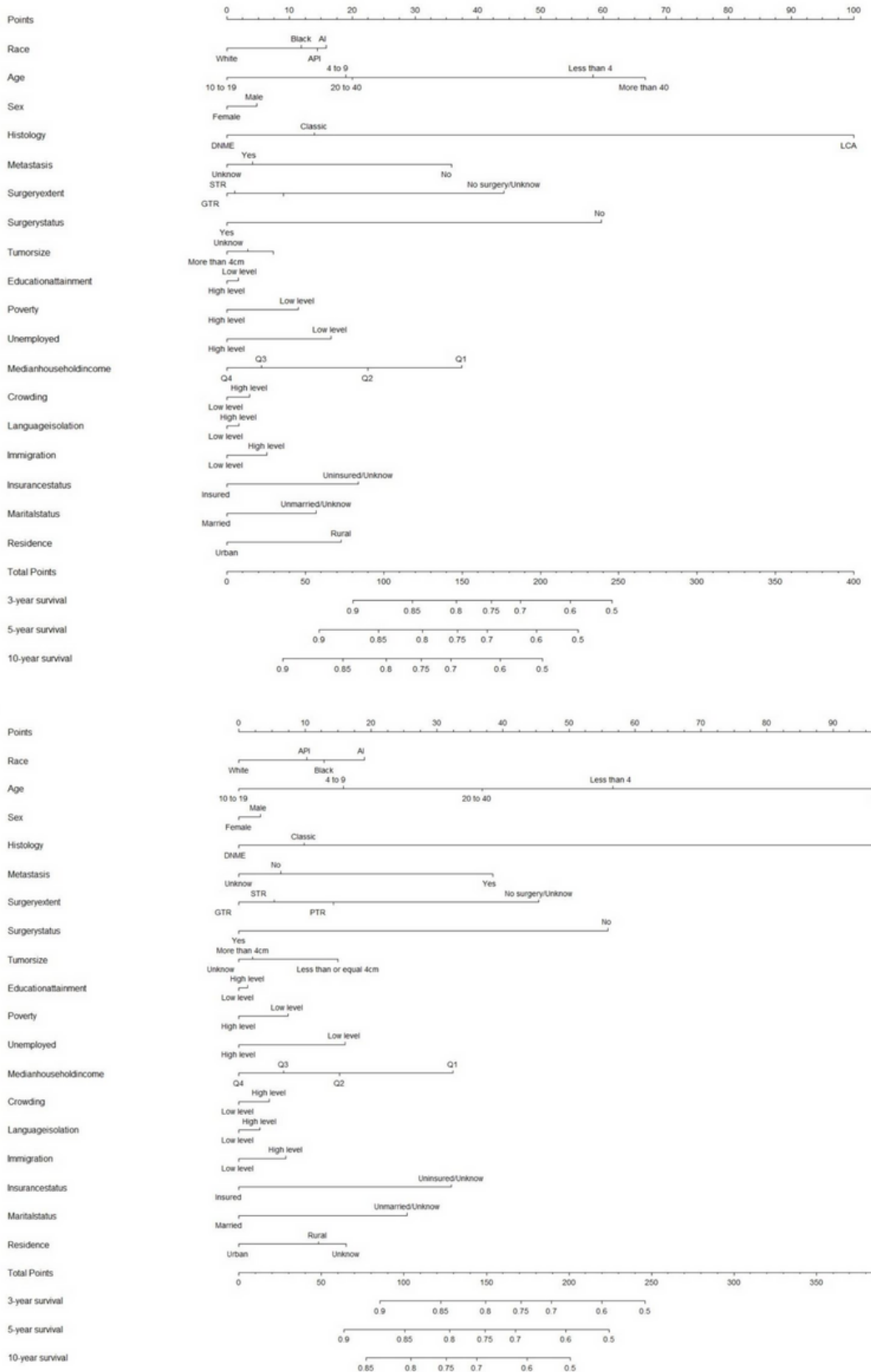

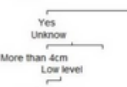

togn evel Los ever

mognever conver

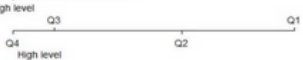

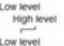

Low Hon

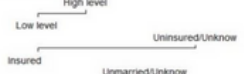

marnos

unson
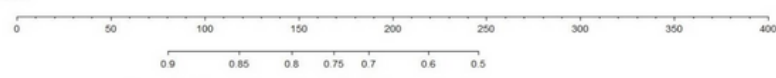

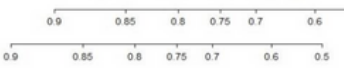
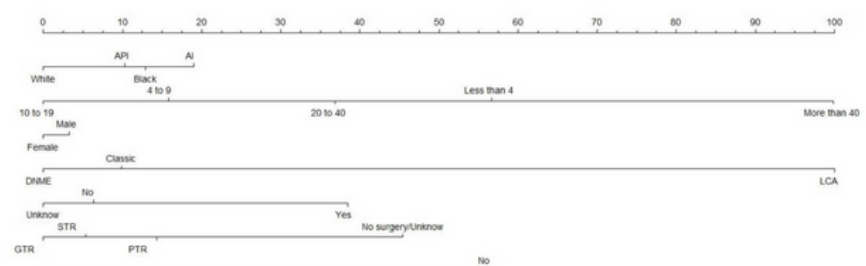

res

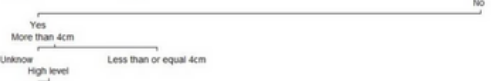

cosionet

tounteret

nonient couseret

${ }_{\text {at }}^{\text {and }}$

non tont

and ived

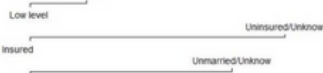

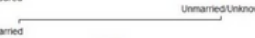

unam

is

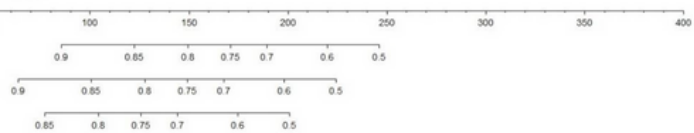

\section{Figure 4}

Prognostic nomograms $(A=M B S D, B=0 S)$ of survival probabilities at 3-, 5-, and 10- years. abbreviation: API, Asian/Pacific Islander; Al, American Indian; DNME, Desmoplastic Nodular Medulloblastoma; LCA, Large Cell Medulloblastoma; PTR, Partial Tumor Resection; STR, Subtotal Tumor Resection; GTR, Gross Tumor Resection; Q, Quartile. 
A
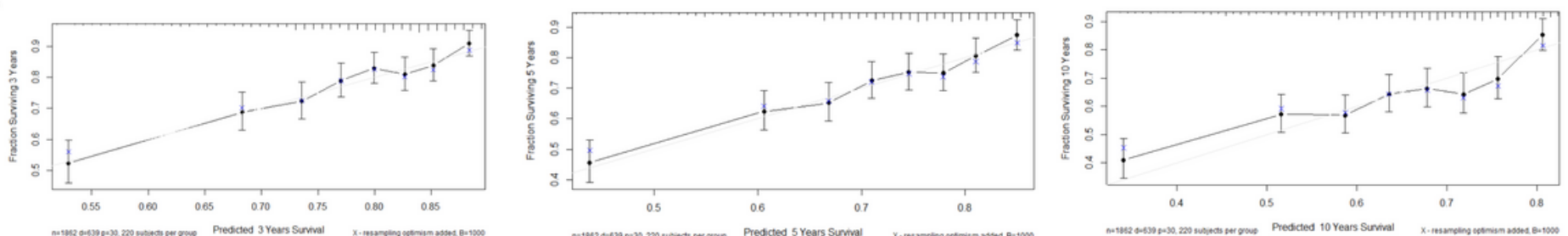

B
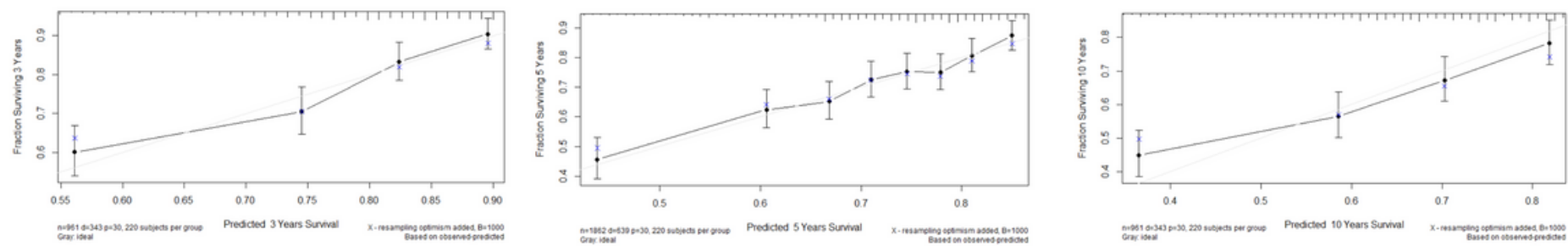

\section{Figure 5}

Calibration plots of MBSD at 3-, 5- and 10-years in train cohort (A) and validation cohort (B).

A
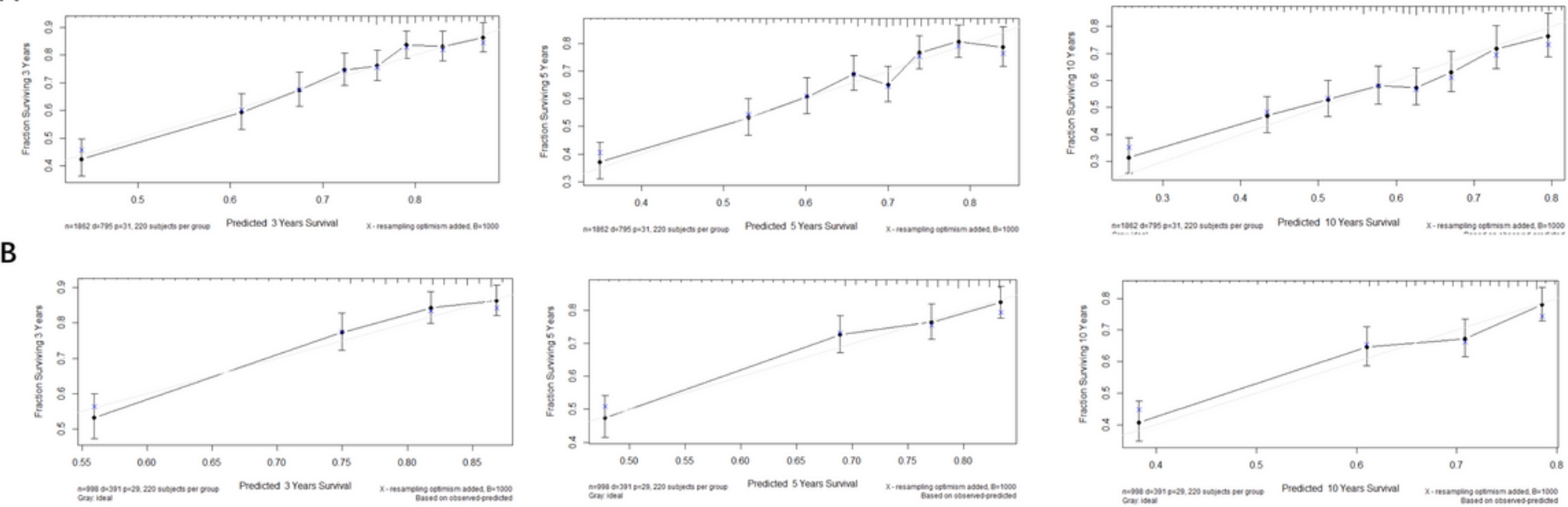

Figure 6

Calibration plots of OS at 3-, 5- and 10-years in train cohort (A) and validation cohort (B).

\section{Supplementary Files}

This is a list of supplementary files associated with this preprint. Click to download.

- SupplementaryMaterialforReview.docx 